\title{
Formação para o trabalho em equipe na residência multiprofissional em saúde
}

\section{Training for teamwork in multidisciplinary residency in health}

\author{
Isis Alexandrina Casanova ${ }^{1}$, Nildo Alves Batista², Lídia Ruiz-Moreno²
}

${ }^{1}$ Programa de Pós-Graduação Interdisciplinar em Ciências da Saúde, Universidade Federal de São Paulo (UNIFESP) - São Paulo (SP), Brasil.

${ }^{2}$ Centro de Desenvolvimento do Ensino Superior em Saúde (CEDESS), UNIFESP - São Paulo (SP), Brasil.

DOI: http://dx.doi.org/10.7322/abcshs.v40i3.800

\section{RESUMO}

Introdução: As mudanças das necessidades de saúde e a implementação do Sistema Único de Saúde (SUS) no Brasil trouxeram à tona a necessidade de discutir e ampliar os debates sobre a atuação dos profissionais para um trabalho em equipe interprofissional. A Educação Interprofissional (EIP) apresenta-se atualmente como estratégia para formar profissionais para o trabalho em equipe, essencial para a integralidade no cuidado. Objetivo: $\mathrm{O}$ objetivo da pesquisa foi analisar a percepção dos profissionais que cursam a Residência Multiprofissional em Saúde (RMS) sobre a formação para o trabalho em equipe. Métodos: Pesquisa de abordagem qualitativa de caráter exploratório descritivo. O instrumento utilizado para a coleta de dados foi uma questão aberta, componente de uma escala atitudinal que investigou a temática do trabalho em equipe. Participaram da pesquisa 76 residentes de segundo ano de duas instituições (uma pública federal e uma estadual) do Estado de São Paulo. Resultados: Os resultados passaram pela análise de conteúdo, tipo temático. A análise temática das respostas obtidas sobre a formação para o trabalho em equipe foi agrupadanas seguintes categorias: atendimento conjunto e tomada de decisão compartilhada no tratamento; trabalho em equipe para a integralidade do cuidado com centralidade no paciente; reconhecimento dos limites e especificidades de cada profissão, bem como a sua integração. Conclusão: Este trabalho demonstrou o potencial da RMS em formar profissionais de saúde para o trabalho em equipe e, consequentemente, para a transformação das práticas, com vistas à integralidade no cuidado.

Palavras-chave: recursos humanos em saúde; educação em saúde; equipe de assistência ao paciente; internato não médico; educação superior.

\begin{abstract}
Introduction: The changing health needs and the implementation of Health System (SUS) in Brazil brought the need to discuss and expand the debate on the role of professionals to work in interprofessional team. The intereducation (EIP) presents itself today as a strategy to train professionals to work in teams, essential for completeness in care. Objective: The objective of the research was to analyze the perception of professionals who attend the Multidisciplinary Residency in Health (RMS) on training for teamwork. Methods: It is a qualitative study of descriptive exploratory character. The instrument used for data collection was an open question, component of an attitudinal scale that investigated the theme of teamwork. The participants were 76 residents of the second year of two institutions (a federal government and a state) of São Paulo. Results: The results have gone through content analysis, thematic type. The thematic analysis of responses obtained on the training for teamwork was grouped into the following categories: service together and shared decision-making in the treatment; Teamwork for comprehensive care of the patient centrality; recognition of the limits and specificity of each profession, and the integration between them. Conclusion: This study demonstrated the potential of RMS to train health professionals to work in a team and hence to the transformation of practices with a view to completeness in care.
\end{abstract}

Keywords: health manpower; health education; patient care team; internship, nonmedical; education, higher. 


\section{INTRODUÇÃO}

As mudanças das necessidades de saúde e a implementação do Sistema Único de Saúde (SUS) no Brasil trouxeram à tona a necessidade de discutir e ampliar os debates sobre a atuação dos profissionais para um trabalho em equipe interprofissional. A tendência dos profissionais de saúde é atuar de forma fragmentada e desvinculada de uma abordagem integral que contemple as múltiplas dimensões das necessidades de saúde dos usuários e da população ${ }^{1}$.

A Educação Interprofissional (EIP) apresenta-se atualmente como uma estratégia para formar profissionais aptos para o trabalho em equipe, prática essencial para a integralidade no cuidado em saúde. A EIP contribui para a formação de profissionais de saúde mais bem preparados para uma atuação integrada em equipe, na qual a colaboração e o reconhecimento da interdependência das áreas predominam adiante da competição e da fragmentação ${ }^{2}$.

A EIP consiste de oportunidades de formação conjuntas para o desenvolvimento de aprendizagens compartilhadas nas quais duas ou mais profissões aprendem juntas com e sobre as outras ${ }^{3}$. Como proposta de formação, a EIP vem sendo discutida nos últimos 30 anos, especialmente nos Estados Unidos e na Europa, com o intuito de estimular o aprimoramento do cuidado em saúde por meio do trabalho de equipe. Os princípios da EIP se aplicam tanto para a graduação das diferentes profissões de saúde quanto para a educação permanente dos profissionais componentes de uma equipe de trabalho ${ }^{3}$.

Entre as estratégias formuladas pelos Ministérios da Educação e da Saúde, destacam-se os programas de Residência Multiprofissional em Saúde (RMS), que têm o objetivo de formar profissionais de saúde, não médicos, com vistas a superar a segmentação do conhecimento e do cuidado na atenção em saúde. Essa modalidade de formação profissional oferece titulação em pós-graduação lato sensu, utilizando-se como estratégia de ensino-aprendizado a formação em serviço (pelo trabalho), mediante acompanhamento e supervisão ${ }^{4}$.

$\mathrm{Na}$ presente pesquisa objetivou-se analisar a percepção dos profissionais que cursam a RMS sobre a formação para o trabalho em equipe.

\section{MÉTODOS}

Este artigo é um recorte de uma pesquisa de doutorado mais ampla relativa à EIP e à RMS. Apresenta uma abordagem qualitativa de caráter exploratório descritivo ${ }^{5}$. O instrumento utilizado para a coleta de dados foi uma questão aberta, componente de uma escala atitudinal que investigou a temática, em que foi perguntado aos residentes: "Como é desenvolvido o trabalho em equipe na RMS?". Participaram da pesquisa 76 residentes de segundo ano de duas instituições (uma pública federal e uma estadual) do Estado de São Paulo. Dos 76 profissionais, 2 (2,07 \%) são homens, e 74 (97,3\%), mulheres. Quanto à faixa etária, 47 (61,84\%) têm menos que 26 anos, 24 (31,57\%), entre 26 e 30 anos, e $5(6,57 \%)$, 31 anos ou mais. Em relação à formação profissional, $10(13,15 \%)$ são assistentes sociais, 1 (1,31\%) é educador físico, 20 (26,31\%) são enfermeiros, 2 (2,63\%),o farmacêuticos, 13 (17,10\%), fisioterapeutas, 5 (6,57\%), fonoaudiólogos, 10 (13,15\%), nutricionistas, 11 (14,47\%), psicólogos, e 4 (5,26\%), terapeutas ocupacionais. Quanto à instituição de origem, 31 (40,78\%) pessoas estudaram em instituições particulares, e 45 (59,21\%), pessoas em públicas. A numeração segue a ordem das respostas dos participantes da pesquisa (E1, E2, E3 etc.). Os resultados passaram pela análise de conteúdo, tipo temática ${ }^{6}$.

O projeto foi aprovado com o parecer $n^{\circ} 429.129 / 2013$ pelo Comitê de Ética em Pesquisas com Seres Humanos da Universidade Federal de São Paulo (UNIFESP) e todos os participantes assinaram um Termo de Consentimento Livre e Esclarecido.

\section{RESULTADOS E DISCUSSÃO}

A análise temática das respostas obtidas sobre a formação para o trabalho em equipe foi agrupada nas seguintes categorias: atendimento conjunto e tomada de decisão compartilhada no tratamento; trabalho em equipe para a integralidade do cuidado com centralidade no paciente; reconhecimento dos limites e especificidades de cada profissão, bem como a sua integração. São apresentadas a seguir essas categorias apreendidas a partir das unidades de contexto, e suas respectivas unidades de registro.

A primeira categoria revela que nas RMSs estudadas os atendimentos são realizados de forma conjunta por parte dos profissionais que integram as equipes nas diferentes etapas da assistência. No cotidiano do serviço, inicialmente o paciente é acolhido e avaliado pelos profissionais das diferentes áreas, quando são discutidas suas necessidades. Posteriormente, é traçado um plano de trabalho com o aporte de cada profissão e, ao final, são realizadas uma avaliação e uma proposta de acompanhamento do caso.

$\mathrm{Na}$ geriatria, sempre que um paciente é internado, todos os profissionais avaliam, posteriormente, compartilhamos nossas avaliações e percepções, e assim construímos um plano de cuidados que engloba paciente, família e cuidados. (E1)

Podemos observar que o trabalho em equipe na RMS viabiliza ações conjuntas em prol do paciente em uma relação recíproca de dupla mão, entre trabalho e interação. Assim, a comunicação entre profissionais faz parte do exercício cotidiano de trabalho e permite articular as inúmeras ações executadas na equipe, no serviço e na rede de atenção ${ }^{7}$.

O trabalho em equipe acontece também no planejamento das ações, na construção dos PTS (plano terapêutico singular), nos atendimentos compartilhados e na aproximação dos profissionais com a realidade do território com posterior troca das percepções. (E8) 
A equipe tem sua própria organização de fazer acolhimento do usuário/família, normalmente com dois profissionais, pois fazer o levantamento das questões e demandas para assim ver quais são as prioridades do usuário/ família e quais são as prioridades que os profissionais identificam e quais são os profissionais que precisam atuar no acompanhamento. Organizamo-nos para serem realizadas reuniões diárias para a discussão dos casos, grupos e ações desenvolvidas pela equipe. (E9)

A valorização do cuidado, o trabalho em equipe interprofissional e a facilidade de desenvolver e aplicar os projetos terapêuticos singulares (PTSs) favorecem as discussões de caso, o desenvolvimento de noções interdisciplinares, as contribuições das várias profissões e de suas especificidades, requerendo uma reflexão mais sistematizada em relação ao cuidado prestado ${ }^{8}$.

O trabalho em equipe desenvolve-se nos espaços de discussão e encontros na prática e também nos contextos de aulas teóricas. A partir disso afinam-se as necessidades da instituição, do paciente e possibilidade de atuação de cada profissional. (E14)

$\mathrm{Na}$ experiência que tive, o trabalho é desenvolvido a partir da inserção dos residentes em equipes da ESF e do NASF do município, da realização de plantões em Pronto Socorro e Pronto Atendimento, aulas teóricas, consultorias, atuação em trabalho junto à comunidade. (E16)

É interessante destacar que dificuldades e desafios para o trabalho em equipe de forma integrada surgem também nas respostas dos residentes:

No segundo ano, as equipes de residentes mantiveram momentos conjuntos, mas sem discussões de equipe, devido a fragmentação do serviço e que nos inserimos (ambulatório de saúde mental), no entanto ocorreram trocas informais sobre casos, grupos, atividades, questões do serviço, etc. (E17)

Os residentes referem ainda um modelo de atenção tradicional (biologicista e hospitalocêntrico) que interfere negativamente no trabalho em equipe de forma integrada. Importante enfatizar que é comum na área da saúde a tendência de profissionais atuarem de forma isolada, dissociada e fragmentada, independente das demais, repercutindo no atendimento final ao paciente e expressando longas formações circunscritas a uma única área de atuação ${ }^{9}$.

O cuidado do paciente deve englobar toda a equipe, objetivando o seu melhor tratamento. Dependendo do setor em que atuamos, o trabalho em equipe não funciona de maneira multiprofissional, seguindo os parâmetros do modelo biomédico e hospitalocêntrico. (E13)
Os residentes percebem também que há diferença entre os atendimentos em conjunto e as discussões, que não ocorrem com tanta facilidade em um determinado serviço devido a sua fragmentação, porém percebem que, por meio da implementação de estratégias que promovam o desenvolvimento do trabalho em equipe, o serviço começa a migrar desse estado fragmentado para outro onde são fortalecidos, melhorando os resultados na saúde e no trabalho em equipe.

Na saúde mental, cenários como os CAPS possibilitam o desenvolvimento de trabalho em equipe e planejamento conjunto de estratégias, porém, em cenários como o ambulatório esse trabalho em equipe fica dificultado pela própria fragmentação de um funcionamento ambulatorial. (E18)

Por outro lado, a falta de integração de algumas equipes é mencionada, o que implica em pouco envolvimento dos profissionais na discussão que permeia o trabalho.

[...] É desenvolvido ainda de forma isolada no sentido de algumas discussões em que seria necessária uma melhor integração da equipe... (E4)

Uma segunda categoria identificada nas respostas dos residentes foi "o trabalho em equipe para a integralidade do cuidado com centralidade no paciente".

É importante destacar que, independente da complexidade do serviço de saúde, o cuidado no trabalho em equipe deve priorizar a atenção individual, sempre atento à possibilidade e à potencialidade de agregação de outros saberes, bem como de outras práticas disponíveis em outros serviços, de saúde ou não ${ }^{10}$.

$\mathrm{O}$ trabalho em equipe com vistas à integralidade do cuidado no espaço singular de cada serviço de saúde é assim definido como o esforço da equipe em traduzir e atender, da melhor forma possível, necessidades, muitas vezes complexas, captadas em sua expressão individual, o que resulta na "integralidade focalizada", esforço de cada um dos trabalhadores e da equipe como um todo. O atendimento de cada profissional deverá manter o seu compromisso com a integralidade, que só será alcançada com o fruto do trabalho solidário da equipe de saúde, com seus múltiplos saberes e práticas ${ }^{10}$.

O trabalho em equipe está sendo focado em cada cuidado que cada profissão deve realizar e não um cuidado compartilhado com todos, dessa forma o trabalho em equipe está dissociado (E6).

O trabalho em equipe sem a devida atenção prestada ao paciente requer uma maior junção do processo de trabalho que segue a linha da integralidade do cuidado, implicando na construção de novos saberes e práticas em saúde ${ }^{10}$.

Muitas vezes o trabalho é desenvolvido sem a clareza de indicadores para darem concretude ao cuidado prestado e parâmetros 
para avaliação da qualidade do serviço ofertado. Faltam espaços e períodos para a reunião da equipe, para a construção de uma assistência conjunta, bem como para permitir maior interação entre os profissionais, discussão de expectativas, necessidades e experiências dos indivíduos. (E12)

Espera-se, assim, que os profissionais de saúde tenham um olhar mais atento a seus pacientes/usuários, não restringindo suas observações à relação saúde-doença, mas interpretando os vários fatores que intervêm nela. No dia a dia, os profissionais não devem fragmentar o cuidado com o paciente ou desvincular sua prática profissional dos demais colegas ${ }^{11}$.

A integralidade do cuidado só pode ser obtida em rede. Pode haver algum grau de integralidade "focalizada" quando uma equipe, em um serviço de saúde, por intermédio de uma boa articulação de suas práticas, consegue escutar e atender, da melhor forma possível, às necessidades de saúde trazida por cada um ${ }^{10}$.

O trabalho de equipe na residência acontece o tempo todo, desde quando aprendemos teoricamente o mínimo de cada profissão necessária para o cuidado integral, até acompanhar as avaliações e intervenções de toda a equipe, discutir todos os casos em conjunto... (E2)

Parte-se do princípio de que se os profissionais de saúde forem capazes de simultaneamente identificar os pontos fortes de cada membro da equipe de assistência e utilizá-los para lidar com os problemas mais complexos do paciente e de toda a família envolvida, desempenharão um papel fundamental e necessário para o cuidado integral.

O trabalho em equipe multiprofissional é desenvolvido com a interação entre os profissionais de saúde devendo haver comunicação e compartilhamento das informações sobre os pacientes para que cada profissional consiga se inserir no cuidado integral. (E5)

O trabalho é desenvolvido de forma mutua cada um respeitando o seu espaço e interagindo com o outro, ajudando e sempre buscando trocas de conhecimento em prol de um atendimento melhor e mais integrado no paciente. (E10)

A integralidade requer a implementação clara e precisa de uma formação comprometida com o desenvolvimento de competências gerais necessárias a todos os profissionais de saúde, tendo em vista uma prática de qualidade que desenvolva a capacidade de análise crítica de contextos e que problematize saberes e processos de educação permanente no desenvolvimento das competências específicas de cada trabalho ${ }^{12}$.

A comunicação entre os residentes é horizontalizada, as decisões são sempre discutidas entre os membros da equipe levando-se em conta o saber de cada profissional/área de atuação. Estas discussões promovem práticas interdisciplinares com foco na integralidade do cuidado. As ações são sempre de corresponsabilidade entre os membros da equipe. (E19)

A terceira categoria identificada foi o "reconhecimento dos limites e especificidades de cada profissão, bem como a sua integração".

[...] Um paciente idoso quase sempre tem demanda para diversos profissionais da saúde, sendo assim, logo reconhecemos nosso limite e percebemos a necessidade da avaliação de outro profissional. Aprendemos a conversar e compartilhar as informações que adquirimos para que assim, juntos, seja possível traçar um plano de cuidados... (E1).

Em miniequipes, sempre estamos juntos e em grande escala de comunicação trabalhando a favor do paciente. Tiramos dúvidas uns dos outros, e muitas vezes aprendemos que podemos ajudar não somente com a nossa função específica da profissão. (E11)

Os membros da equipe devem preservar suas funções específicas e manter a comunicação uns com os outros, colocando-se como responsáveis, principalmente nos trabalhos que envolvem a geriatria ${ }^{13}$.

Entretanto, um dos residentes destaca dificuldades na construção de sua identidade profissional:

[...] Um ponto negativo é que sinto que não tenho uma formação específica (teórico-prática) tão aprofundada, uma vez que a ênfase é dada mais ao caráter global/ geral da interdisciplinaridade... (E3)

A formação para o trabalho em equipe não compromete a identidade profissional, na realidade reforça; o trabalho de equipe, a discussão de papéis profissionais, o compromisso na solução de problemas e a negociação na tomada de decisão são características marcantes. Para isso, a valorização da história de diferentes áreas profissionais e a consideração do outro como parceiro legítimo na construção de conhecimentos, com respeito pelas diferenças em um movimento de busca, diálogo, desafio, comprometimento e responsabilidade, são componentes essenciais para o desenvolvimento do trabalho em equipe na RMS ${ }^{2}$.

Conclui-se que os dados obtidos com os 76 participantes da pesquisa, especificamente de duas instituições públicas do Estado de São Paulo, mostram o potencial da RMS em formar profissionais de saúde para o trabalho em equipe e, consequentemente, para a transformação das práticas, com vistas à integralidade no cuidado.

De acordo com as categorias apreendidas, a primeira enfatiza que os atendimentos são realizados de forma conjunta 
entre os profissionais da equipe, permitindo um melhor planejamento das ações e o estabelecimento de um plano terapêutico singular.

A segunda categoria refere-se ao preparo para o trabalho em equipe para a integralidade do cuidado com centralidade no paciente. Observamos que este trabalho segue na linha do cuidado integral prestado ao paciente, com um olhar mais atento e sem se restringir ao processo saúde/doença. Por fim, a última categoria identificada reconhece os limites e as especificidades de cada profissão. Verificamos que durante o trabalho em equipe os profissionais conseguem compartilhar e aprender entre si, preservando suas especificidades e as do outro.

\section{REFERÊNCIAS}

1. Peduzzi M, Norman IJ, Germani ACCG, Silva JAM, Souza GC. Educação Interprofissional: formação de profissionais de saúde para o trabalho em equipe com foco nos usuários. Rev Esc Enferm USP. 2013;47(4):977-83 http://dx.doi.org/10.1590/S0080-623420130000400029

2. Batista NA. Educação Inteprofissional em saúde: concepções e práticas. Cad Fenepas. 2012;2:5-28.

3. Worl Health Organization (WHO). World Health Report 2010: Working Together for Health. Geneva: World Health Organization; 2010.

4. Brasil. Ministério da Saúde. Secretaria de Gestão do Trabalho e da Educação na Saúde. Departamento de Gestão da Educação na Saúde. Residência multiprofissional em saúde: experiências, avanços e desafios. Brasília: Ministério da Saúde; 2006. p.414.

5. Gil AC. Como elaborar projetos de pesquisa. $4^{a}$ ed. São Paulo: Atlas; 2002.

6. Franco MLPB. Análise do Conteúdo. 2a ed. Brasília: Líber; 2005.

7. Peduzzi, M. Equipe multiprofissional de saúde: conceito e tipologia. Rev Saúde Pública. 2001;35(1):103-109. http://dx.doi.org/10.1590/S0034-89102001000100016

8. Campos GWS, Amaral MA. A clínica ampliada e compartilhada, a gestão democrática e redes de atenção como referenciais teórico-operacionais para a reforma do hospital. Ciênc Saúde Coletiva. 2007;12(4):849-59.

http://dx.doi.org/10.1590/S1413-81232007000400007

9. Feuerwerker LM. Modelos tecnoassistenciais, gestão e organização do trabalho em saúde: nada é indiferente no processo de luta para a consolidação do SUS. Interface. 2005;9(18):489-506.

http://dx.doi.org/10.1590/\$1414-32832005000300003

10. Cecílio LCO. As necessidades de saúde como conceito estruturante na luta pela integralidade e equidade na atenção à saúde. In: Pinheiro R, Mattos RA. Os sentidos da integralidade na atenção e no cuidado à saúde. Rio de Janeiro: IMS/ABRASCO: 2001. p.113-26.

11. AssumpçãoPFS, MiotoRCT. Revisitando o conceito de integralidade emsaúdeapartir dateoriacrítico dialética. Disponível em:http://www. joinpp.ufma.br/jornadas/joinpp/V/eixos/9_estados-e-lutas-sociais/ revisitando-o-conceito-de-integralidade-em-saude-a-partir-dateoria-critico-dialetica.pdf. Acesso 15 jul. 2015.

12. Brasil. Ministério da Educação. Conselho Nacional da Educação. Parecer CNE/CES 1133/2001. Disponível em: http://portal.mec. gov.br/dmdocuments/ces1133.pdf Acesso em: 16 jul. 2015.

13. Pinho MCG. Trabalho em equipe de saúde: limites e possibilidades de atuação eficaz. Ciênc Cognição. 2006;8:68-87. 